\title{
Development of Ultrashort Pulsed VUV Laser and its Applications
}

\author{
Masahito Katto ${ }^{1}$, Masanori $\mathrm{Kaku}^{2}$, Atsushi Yokotani ${ }^{2}, \mathrm{Kenzo} \mathrm{Miyazaki}^{3}$, Noriaki Miyanaga ${ }^{4}$, and Shoichi Kubodera ${ }^{2}$ \\ ${ }^{1}$ Center for Collaborative Research and Community Cooperation, Photon Science Project, University of Miyazaki, \\ 1-1 Gakuen-Kibanadai-Nishi, Miyazaki 889-2192, Japan \\ E-mail: mkatto@opt.miyazaki-u.ac.jp \\ ${ }^{2}$ Faculty of Engineering, Photon Science Project, University of Miyazaki, 1-1 Gakuen-Kibanadai- \\ Nishi, Miyazaki 889-2192, Japan \\ ${ }^{3}$ Institute of Advanced Energy, Kyoto University, Gokasho, Uji, Kyoto 611-0011, Japan \\ ${ }^{4}$ Institute of Laser Engineering, Osaka University, 2-6 Yamadaoka, Suita, Osaka 565-0871, Japan
}

\begin{abstract}
Vacuum ultraviolet (VUV) laser systems for advanced applications such as micro and precise processing and photochemical reactions have been developed. We have been constructing a VUVMOPA system to generate output energy of sub-mJ with a pulse width of sub-picosecond at the wavelength of $126 \mathrm{~nm}$. A VUV seed pulse, generated by the 7th harmonics of a $882 \mathrm{~nm}$ Ti:Sapphire (TiS) laser was amplified by the $\mathrm{Ar}_{2}{ }^{*}$ medium generated from an optical-field-induced ionization of Ar plasma pumped by the TiS laser. We achieved an amplification of $126 \mathrm{~nm}$ seed pulse by a factor of 2.6. Recently, we introduced a new $800 \mathrm{~nm}$ TiS laser. This laser was synchronized with the 882 $\mathrm{nm}$ TiS laser and the delay between these outputs was controllable. We also developed a photostimulated desorption spectroscopy method for surface analysis. A tunable incoherent VUV radiation obtained from a laser induced plasma was irradiated the samples and then desorbed species were analyzed by mass spectroscopy. This method enables analysis without sample heating and damaging.

DOI: $10.2961 /$ jlmn.2014.02.0006
\end{abstract}

Keywords: vacuum ultraviolet, harmonics, excimer, ultra-short pulse, MOPA system, application

\section{Introduction}

In the vacuum ultraviolet (VUV) spectral region, corresponding to the wavelength range from 100 to $200 \mathrm{~nm}$, the photon has energy around $10 \mathrm{eV}$, which is above the bandgap energy of almost all materials. VUV radiations are thus strongly absorbed and can excite the materials surface efficiently. Then, the material processing using VUV photon is promising technology as an environmental friendly process that requires neither catalyst nor solvent, since the excitation by the high-energy VUV photons itself induces the surface reaction. The VUV radiations, especially coherent light sources, are thus in high demand for advanced precise and microscopic processing.

In the VUV region, $F_{2}$ and ArF excimer lasers were lasing by discharge pumping at the wavelength of $157 \mathrm{~nm}$ and $193 \mathrm{~nm}$, respectively. Rare gas excimers, $\mathrm{Xe}_{2}{ }^{*}, \mathrm{Kr}_{2}{ }^{*}$ and $\mathrm{Ar}_{2}{ }^{*}$, pumped by e-beams and discharges also emit the VUV radiations at the wavelengths of $172 \mathrm{~nm}, 146 \mathrm{~nm}$ and $126 \mathrm{~nm}$, respectively. We have been studying about the rare gas excimer medium for high intense VUV light sources. We have already developed an e-beam pumped rare gas excimer laser emitting the output energy of 16 $\mathrm{MW}$ at the wavelength of $126 \mathrm{~nm}[1,2]$ from $\mathrm{Ar}_{2}{ }^{*}$ and 6.6 $\mathrm{MW}$ at the wavelength of $146 \mathrm{~nm}$ [3] from $\mathrm{Kr}_{2}{ }^{*}$. We also developed a discharge pumped $\mathrm{Kr}_{2}{ }^{*}$ laser and successfully obtained output energy of $100 \mu \mathrm{J}$ [4]. Until now, we could achieve high power ns-pulsed VUV laser only by the ebeam pumping system, which was needed a huge electric system and had a low repetition rate, approximately $30 \mathrm{~min}$ per shot. Another approach was to develop VUV excimer lamps emitting incoherent quasi-continuous wave VUV light. These lamps emitted the VUV photons originated from the rare gas excimers pumped by the silent discharge. We also have developed the rare gas excimer lamps and demonstrated the VUV processing by using these lamps [5]. The excimer lamp was very convenient, however its output is incoherent and the power may not be always high enough, resulting slow process rate. A high-intense coherent VUV source, for example having a sub-mJ energy and sub-picosecond pulsed output at a high repetition rate, should be desired from the viewpoint of applications.

Ultrashort-pulsed radiations in the femtosecond (fs) region are also attractive especially for materials processing. These pulses can ablate materials with less heat effects compared to nanosecond laser. Cutting and scribing with fs lasers should become one of the targets for advanced materials processing. From a viewpoint of novel precise laser processing, an ultrashort-pulsed VUV laser should become of great interest, since they should simultaneously optimize the heat effects of materials and the interaction at very shallow surfaces of them. Very few ultrashort-pulsed VUV lasers, however, have been developed [6-9].

We have been developing a new VUV laser system for sub-picosecond pulse [10] at the wavelength of $126 \mathrm{~nm}$ and repetition rate of above $10 \mathrm{~Hz}$, containing VUV harmonic generation of near-infrared (NIR) pulsed laser [11] and VUV $\mathrm{Ar}_{2}{ }^{*}$ amplifier system using the optical-field-induced ionization (OFI) plasma [12-14] excited by NIR-fs laser. Recently, we have demonstrated that rare gas excimers 
generated by OFI plasma efficiently amplified the VUV pulse.

We also developed a photon-stimulated desorption (PDS) spectroscopy using a radiation from ultraviolet (UV) to extreme UV (EUV) emitted from Ar plasma [15]. The ultrashort-pulsed VUV laser should be applicable not only for the material processing, such as cutting, drilling and surface alteration not also for the surface analysis with both spatial and time resolution.

In this paper, we describe the presenting results of our VUV laser development at wavelength of $126 \mathrm{~nm}$ and also introduce a surface analysis method, PDS, as an example of the application of VUV radiations.

\section{VUV laser development}

We developed a VUV laser system with subpicosecond pulse width and sub-mJ output energy at wavelength of $126 \mathrm{~nm}$. This system was master-oscillator power amplification (MOPA) system including a VUV oscillator at $126 \mathrm{~nm}$ by harmonic generation and a amplifier by OFI plasma produced $\mathrm{Ar}_{2}{ }^{*}$ medium.

\subsection{VUV oscillator}

For VUV seed pulse generation by harmonic conversion, we used an ultrashort pulsed Ti:Sapphire (TiS) laser (Spectra Physics, Tsunami and Spitfire Pro), which produced a linearly polarized output at wavelength of $882 \mathrm{~nm}$ with a pulse energy up to $1 \mathrm{~mJ}$ and a pulse width of around $160 \mathrm{fs}(\mathrm{FWHM})$ at a repetition rate of $1 \mathrm{kHz}$. The TiS laser output was focused into a gas chamber by using a lens with a focal length of $50 \mathrm{~cm}$. The maximum laser intensity in vacuum was about $10^{14} \mathrm{~W} / \mathrm{cm}^{2}$. We examined maximum output of 7th harmonics using $\mathrm{He}, \mathrm{Ne}, \mathrm{Ar}, \mathrm{Kr}$ and $\mathrm{Xe}$ gases and changing the pressure. Then, he $7^{\text {th }}$ harmonic emission of the $882 \mathrm{~nm}$-TiS laser was optimized as VUV seed pulse at $126 \mathrm{~nm}$ in the Xe gas at pressure of 1.2 Torr [11].

\subsection{VUV amplifier}

We also studied the characteristics of $\mathrm{Ar}_{2}{ }^{*}$ medium

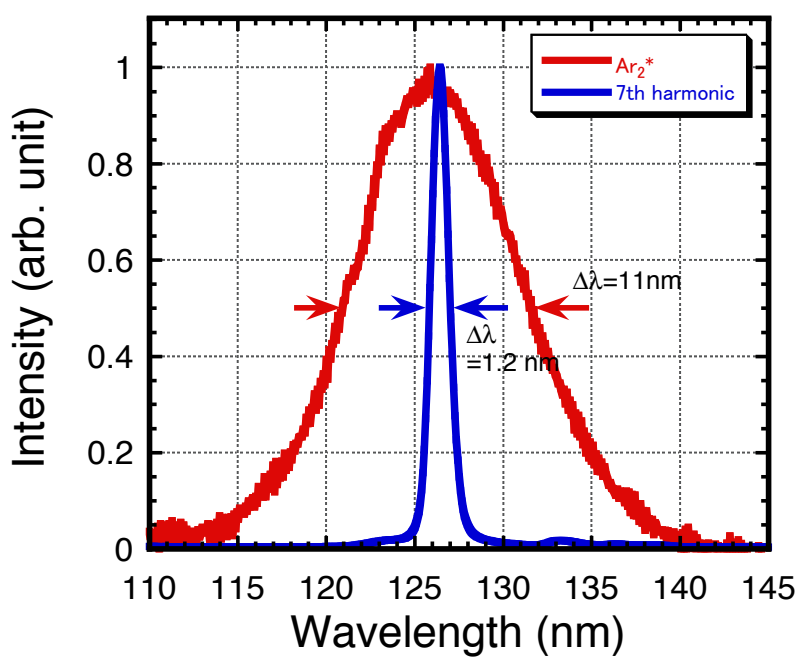

Fig. 1 7th harmonic spectrum generated in Xe by $882 \mathrm{~nm}$ TiS laser and $\mathrm{Ar}_{2}{ }^{*}$ emission spectrum produced by OFI plasma pumped by $800 \mathrm{~nm}$-TiS laser. generated by the OFI plasma pumped by the TiS laser for VUV amplification. Another TiS laser (Thales Laser, Bright) output at wavelength of $800 \mathrm{~nm}$ with a pulse energy of $1 \mathrm{~mJ}$ and a pulse width of $100 \mathrm{fs}$ was focused into a Ar gas at a pressure of around $10 \mathrm{~atm}$. The intensity was about $10^{15} \mathrm{~W} / \mathrm{cm}^{2}$. The Ar gas was excited and ionized by the high intense optical field of the TiS laser and then the Ar plasma was produced and then relaxed to make an excimer $\left(\mathrm{Ar}_{2}{ }^{*}\right)$ state. As a result, we obtained an optical gain of 0.84 $\mathrm{cm}^{-1}[14]$.

In Fig. 1, emission spectra of both VUV seed pulse of $7^{\text {th }}$ harmonic radiation of $882 \mathrm{~nm}$ and OFI produced $\mathrm{Ar}_{2}{ }^{*}$. $\mathrm{The} \mathrm{Ar}_{2}{ }^{*}$ emission had a bandwidth of $11 \mathrm{~nm}$ (FWHM), which was much wider than that of the $7^{\text {th }}$ harmonic of 1.2 $\mathrm{nm}$ (FWHM). We concluded that OFI produced $\mathrm{Ar}_{2}{ }^{*}$ medium should be able to amplify the VUV seed pulse.

\subsection{VUV amplification}

We constructed a VUV-MOPA system, contains seed pulse generator of harmonic conversion and VUV amplifier of OFI produced $\mathrm{Ar}_{2}{ }^{*}$ medium using one TiS laser operated at $882 \mathrm{~nm}$ as shown in Fig. 2. TiS laser produced a pulsed output energy of $1 \mathrm{~mJ}$ with a pulse width of $160 \mathrm{fs}$ at repetition rate of $1 \mathrm{kHz}$. The laser output was split into two beams by a 50:50 beam splitter. One beam was focused into the gas chamber filled with Ar gas at a pressure of 1 $\mathrm{MPa}$ to produce $\mathrm{Ar}_{2}{ }^{*}$ gain medium. The other beam was introduced to the seed pulse generator filled with Xe gas at pressure of around $1 \mathrm{kPa}$ to produce a $7^{\text {th }}$ harmonic for VUV seed pulse through the optical delay line. Optical delay was set to $20 \mathrm{~ns}$ due to the rise time of $\mathrm{Ar}_{2}{ }^{*}$. The fs VUV seed pulse was focused into the gain region of the $\mathrm{Ar}_{2}{ }^{*}$ amplifier using a $\mathrm{MgF}_{2}$ lens. A spatial overlap between the gain region of the amplifier and the fs VUV seed pulse was optimized by adjusting a lens located at the right hand side that was mounted on a two axis linear stage. The amplified fs VUV pulse was separated from the exciting NIR pulse by a dichroic mirror and was reflected to a VUV spectrometer. The VUV emissions were detected as timeintegrated emission spectra using a micro-channel plate (MCP) coupled with the flat-field VUV spectrometer. A linear CMOS sensor was used to detect the visible fluorescence intensity from a phosphor screen that was placed behind the MCP.

We observed the optical amplification of the fs VUV seed pulse at $126 \mathrm{~nm}$ using the OFI $\mathrm{Ar}_{2}{ }^{*}$ amplifier. In Fig. 3, we showed the emission spectra of VUV radiations with and without the $\mathrm{Ar}_{2}{ }^{*}$ amplifier. The VUV seed pulse was amplified by the factor of aournd 2. The spatial overlap between the fs VUV seed pulse and the OFI $\mathrm{Ar}_{2}{ }^{*}$ amplifier was optimized. Figure 4 shows the spatial distribution of the amplification ratio. The amplification ratio was defined as $I_{\text {amp }} / I_{0}$. Here, the Iamp and I0 were the $7^{\text {th }}$ harmonic intensities at $126 \mathrm{~nm}$ with and without the $\mathrm{Ar}_{2}{ }^{*}$ amplification, respectively. The horizontal axis $\mathrm{X}$ represents a displacement of the OFI $\mathrm{Ar}_{2}{ }^{*}$ position, namely the gain region, which was controlled by moving a $\mathrm{MgF} 2$ lens. The spatially-resolved maximum amplification ratio of 2.6 was observed, which corresponded to the one-pass optical gain of $0.94 \mathrm{~cm}^{-1}$. This gain value was consistent with those observed in our previous experiment [14]. A blue curve represents a fitting curve using a Gaussian 


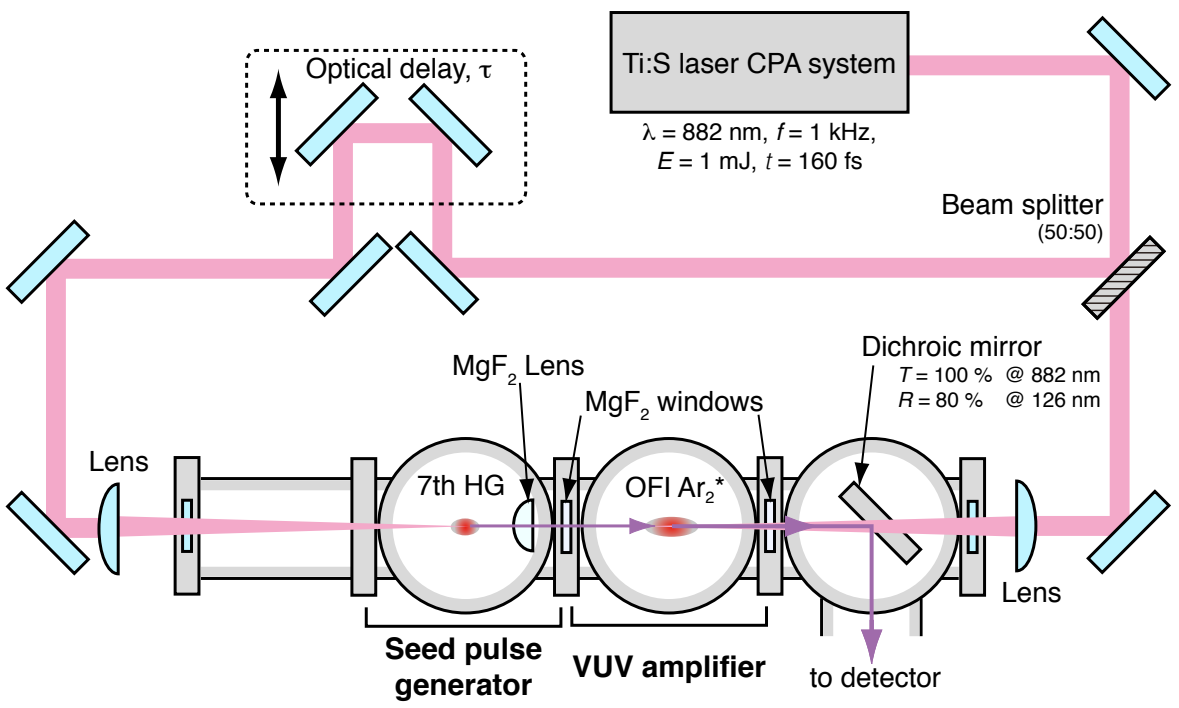

Fig.2 VUV-126 nm MOPA system using one TiS laser.

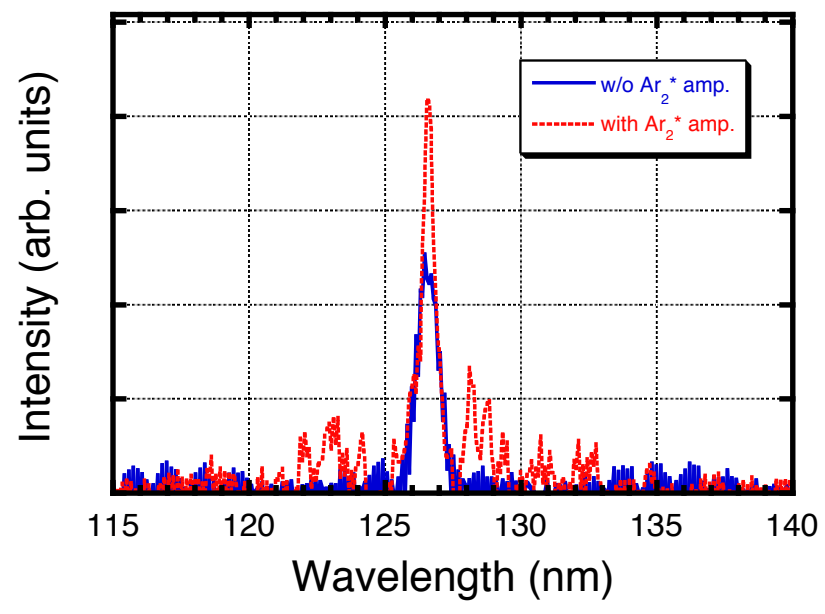

Fig. 3 Emission spectra with and without OFI excited $\mathrm{Ar}_{2}{ }^{*}$ amplifier

function. The gain distribution width of $250 \mu \mathrm{m}$ (FWHM) was observed. Since the size of the VUV seed beam was calculated to be $110 \mu \mathrm{m}$ (FWHM), the deconvoluted gain distribution size was evaluated to be $220 \mu \mathrm{m}$ (FWHM). The initial gain size of $120 \mu \mathrm{m}$ (FWHM) was assumed, which should be same as the size of the focus of the plasma-initiating Ti:Sapphire laser. Considering the gain size difference during the delay time of $20 \mathrm{~ns}$, the average plasma expansion temperature of $1.2 \mathrm{eV}$ was evaluated, which was consistent with our previous result [12].

\subsection{New VUV-MOPA system}

We successfully observed the amplification of VUV seed pulse of $7^{\text {th }}$ harmonic at $882 \mathrm{~nm}$ from NIR-TiS laser using OFI-plasma produced $\mathrm{Ar}_{2}{ }^{*}$ gain medium at repetition rate of $1 \mathrm{kHz}$. The amplification factor was 2.57, which was not enough for the application such as material processing and photochemistry.

Then we introduced a new TiS laser (Spectra Physics, Tsunami and Spitfire Ace) with pulse energy of $5 \mathrm{~mJ}$ with a pulse width of around $120 \mathrm{fs}$ (FWHM) at a repetition rate of $1 \mathrm{kHz}$ for $\mathrm{Ar}_{2}{ }^{*}$ amplifier pumping. The VUV oscillator

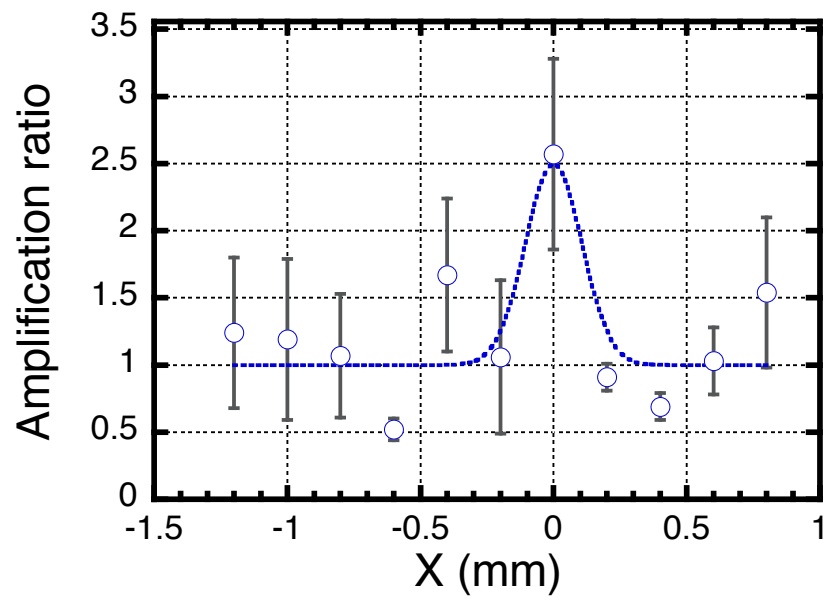

Fig. 4 Spatial distribution of the amplification ratio of the $126 \mathrm{~nm}$ emission.

pulse was generated by $882 \mathrm{~nm}$ TiS laser and the seed pulse should be high since the full output energy can contribute the VUV seed generation. These two lasers were electrically controlled and synchronized with a time jitter of $2 \mathrm{~ns}$. We have been constructing a new MOPA system as shown in Fig. 5.

\section{Application of VUV radiation for surface analysis}

We proposed a new surface analysis technique, mass spectroscopy using photo-simulated desorption (PSD) by a vacuum ultraviolet (VUV) radiation. In this PSD method, the contaminations on the sample surface, e.g. organic materials are desorbed and decomposed by the photochemical effect induced by the high-energy VUV photons. The desorbed species were detected by the mass spectrometer. The PSD enable us to analyze the surface even under the room temperature and is also useful for analyzing polymer substrate. The PSD should meet the needs not only for semiconductor fabrication but also for flexible-display manufacturing. 


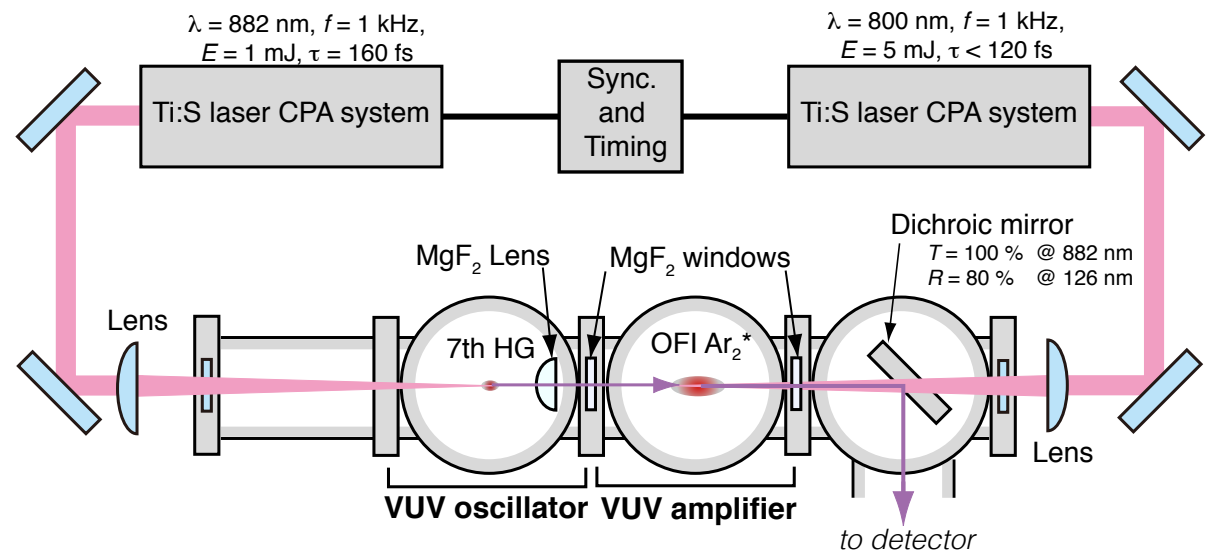

Fig. 5 New VUV MOPA system with two synchronized TiS lasers .

We first experimentally studied about the decomposition process induced by VUV excimer lamps [15]. The results showed the chemical structure should affect the absorption cross-section spectra in this VUV region and then it made difference in the decomposition process. It concluded that we can analyze the surface contamination by examining desorption and decomposition process induced by the VUV radiations. If we use a tunable VUV

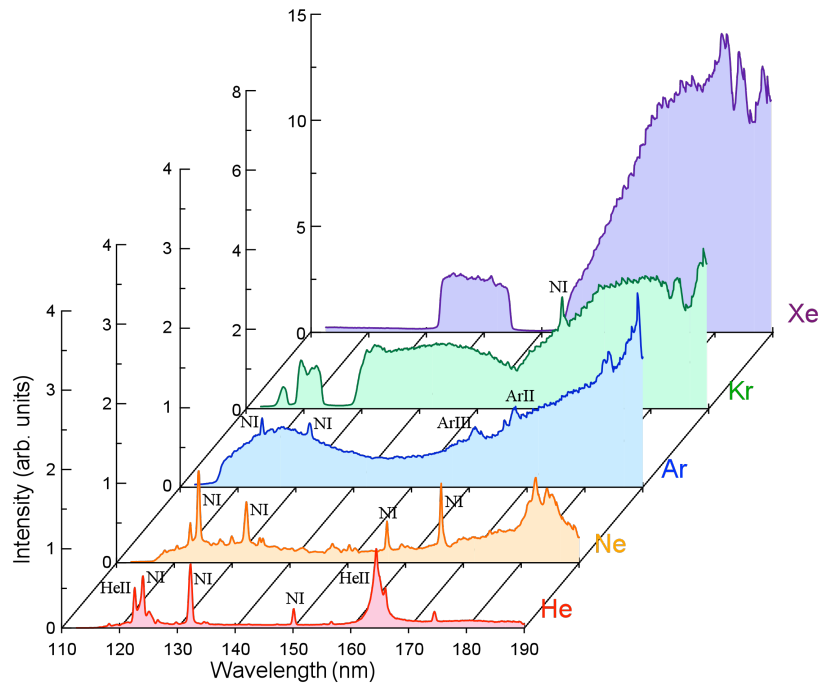

Fig. 6 VUV emission spectra from Nd:YAG laser produced plasma in rare gases. radiation and quantified the decomposition data for several organic materials, we could analyze and identify the contamination materials.

In order to generate a tunable VUV radiation for the surface analysis, we used the laser plasma source. In Fig. 6, we shows the emission spectra of the laser produced plasma in rare gases, $\mathrm{He}, \mathrm{Ne}, \mathrm{Ar}, \mathrm{Kr}$ and $\mathrm{Xe}$. The fundamental output $(\lambda=1064 \mathrm{~nm})$ from Q-switched Nd:YAG laser was focused into the rare gas at the pressure of $0.1 \mathrm{MPa}$ with the focused intensity of $10^{11} \mathrm{~W} / \mathrm{cm}^{2}$. The emission spectra were observed with a VUV spectrometer through a $\mathrm{MgF}_{2}$ window. It is noted here that the short-wavelength cut-off was limited by the transmittance of $\mathrm{MgF}_{2}$ window around $100 \mathrm{~nm}$. We found and concluded that the Ar plasma was suitable source for the high power and broadband VUV radiation.

Figure 7 shows the schematic drawing and appearance picture of our photo-stimulated desorption mass spectrometer (PSD) system [15]. The fundamental output of Qswitched Nd:YAG laser was focused into the VUV-source chamber filled with the Ar gas at the pressure of $0.04 \mathrm{MPa}$. A broadband VUV emission from the Ar plasma was introduced to the VUV monochromator and obtained monochromatic VUV radiation. The wavelength of the radiation was scanned by changing the angle of the grating controlled by the personal computer (PC). The sample was introduced to the main analysis chamber through the load lock chamber. In the main chamber, a tuned VUV radiation was irradiated on the sample surface through the $\mathrm{MgF}_{2}$

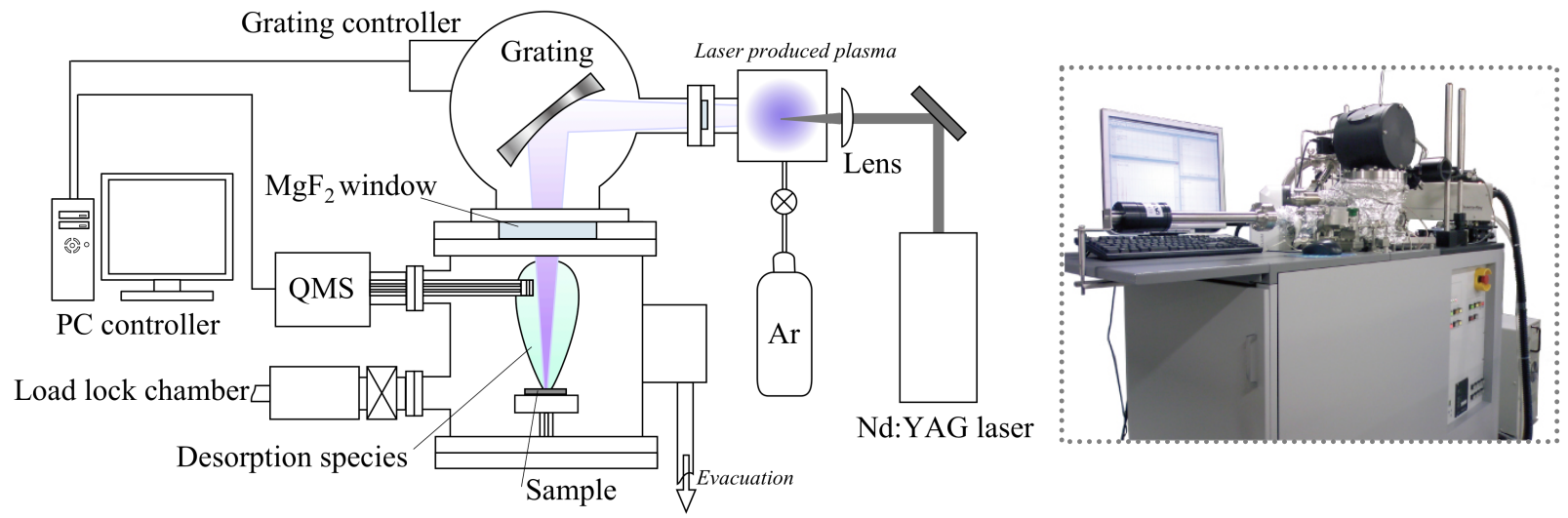

Fig. 7 Schematic drawing and appearance picture Photo-Stimulated Desorption (PSD) mass spectrometer. 


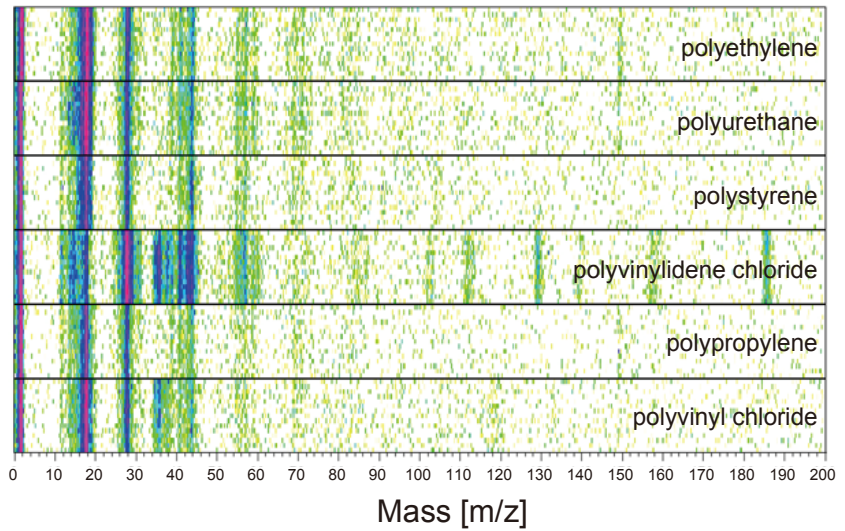

Fig. 8 Mass data of fragments desorped from plastics irradiated by VUV photons at $125 \mathrm{~nm}(\Delta \lambda= \pm 5 \mathrm{~nm})$

window. The shortest wavelength was limited by the $\mathrm{MgF}_{2}$ transmittance to about $100 \mathrm{~nm}$. Materials on the surface strongly absorbed the VUV radiation and then desorbed. A desorbed species was analyzed the quadrupole mass spectrometer (QMS) and its data was stored in the PC.

Figure 8 shows the mass signal spectra obtained from 6 kinds of plastics, polyethylene, polyurethane, polystyrene, polyvinylidene chloride, polypropylene and polyvinyl chloride when the VUV radiation at center wavelength of 125 $\mathrm{nm}$ with the spectral width of $\pm 5 \mathrm{~nm}$ was irradiated. These mass spectra differ from each other. If we accumulate the quantitative spectral data and verify it, it should be possible to identify the material.

\section{Conclusions and Perspectives}

We have been developing VUV radiations and successfully amplified the VUV seed pulse by OFI plasma produced $\mathrm{Ar}_{2}{ }^{*}$ medium. However, the gain was low and the output still low for the application use. A new MOPA system should provide a high power output of VUV seed pulse and a high gain of $\mathrm{Ar}_{2}{ }^{*}$ medium.

Surface analysis by VUV photons is a candidate as a novel method for science and manufacturing. High power coherent VUV radiation in fs regime enables us to analyze the surface with both spatial and time resolution.

\section{Acknowledgments}

The work about VUV laser development was supported by the Grant-in-Aid for Scientific Research (B) Program, the
Grant-in-Aid for Exploratory Research Program and Advanced Research Driving Program (University Collaboration) of MEXT, Japan. The authors also thank NTP, Inc and Hamamatsu Photonics K. K., Japan for their financial support. The PSD works were supported by the collaboration program between the industry and university aided by Ministry of Economy, Trade and Industry, Japan and supported by the Grant-in-Aid for Scientific Research (B) Program The authors thank NTP, Inc and ESCO Ltd.

\section{References}

[1] K. Kurosawa, W. Sasaki, M. Okuda, Y. Takigawa, K. Yoshida, E. Fujiwara, and Y. Kato, Rev. Sci. Instrum., 61, (1990) 728.

[2] K. Kurosawa, Y. Takigawa, W. Sasaki, M. Okuda, K. Yoshida, and Y. Kato, IEEE J. Quantum Electron., QE-27, (1991) 71.

[3] K. Kurosawa, W. Sasaki, E. Fujiwara, and Y. Kato, IEEE J. Quantum Electron., QE-24, (1988) 1908.

[4] W. Sasaki, T. Shirai, S. Kubodera, J. Kawanaka, and T. Igarashi, Opt. Lett., 26, (2001) 503

[5] T. Ohtsubo, M. Takaura, T. Azuma, T. Higashiguchi, S. Kubodera, and W. Sasaki, Appl. Phys., A 76, (2003) 139.

[6] S. P. Le Blanc, Z. Qi, and R. Sauerbrey, Appl. Phys. B, $61,(1995) 439$.

[7] J. Kuntzner and H. Zacharias, Appl. Phys B 66 (1998) 571.

[8] P. Tzankov, O. Steinkellner, J. Zheng, M. Mero, W. Freyer, A. Husakou, I. Babushkin, J. Herrmann, and F. Noack, Opt. Exp., 15, (2007) 6389.

[9] K. Kosma, S. A. Trushin, W. E. Schmid, and W. Fuß, Opt. Lett., 33, (2008) 723.

[10] S. Kubodera, Y. Taniguchi, A. Hosotani, M. Katto, A. Yokotani, N. Miyanaga, and K. Mima, Proc. SPIE, 6452, (2007) 645216.

[11] M. Katto, K. Oda, M. Kaku, A. Yokotani, S. Kubodera, N. Miyanaga, and K. Mima, Opt. Commun., 283, (2010) 414.

[12] M. Kaku, T. Higashiguchi, S. Kubodera, and W. Sasaki, Phys. Rev. A, 68, (2003) 023803.

[13] Y. Morita, T. Higashiguchi, and S. Kubodera, Appl. Phys. B, 82, (2006) 31.

[14] M. Kaku, S. Harano, R. Matsumoto, M. Katto, and S. Kubodera, Opt. Lett., 36, (2011) 2719.

[15] M. Wasamoto M. Katto M. Kaku, S. Kubodera and A. Yokotani, Appl. Surf. Sci., 255, (2009) 9861

(Received: August 26, 2013, Accepted: April 3, 2014) 